\title{
Treatment of Influenza: Prospects of Post-transcriptional Gene Silencing Through Synthetic siRNAs
}

\author{
Oluwagbenga A. Adeola* \\ Department of Medical Microbiology and Parasitology, College of Medicine, Bingham University, Karu, via Abuja, Nigeria; and Centre \\ for Control and Prevention of Zoonoses (CCPZ), University of Ibadan, Ibadan, Nigeria
}

\begin{abstract}
Influenza continues to pose significant threats to global health. The disease remains life-threatening, especially in children, the elderly and immunocompromised patients. High mutation rate and genetic reassortment enhance antigenic diversity and generation of novel strains. These, and other related factors, have made influenza viruses more resistant to control strategies and have necessitated the need for development of novel and effective strategies for prevention and treatment of influenza. While vaccination is the preferred method for prevention of influenza virus infections, preparation of influenza vaccines has to be carried out annually, and this takes several months. Anti-influenza drugs would, therefore, be the most immediate resource for combating newly emerging influenza viruses, especially if available vaccines proved ineffective. This review discusses the prospects of using small interfering RNAs (siRNAs) for treatment of influenza. Challenges associated with anti-influenza RNA interference (RNAi) and future directions are also highlighted
\end{abstract}

\section{Introduction}

Influenza viruses belong to the family Orthomyxoviridae, which has more than five genera. These genera include influenza virus, thogotovirus, and isavirus. ${ }^{1,2}$ There are four types of influenza viruses: A, B, C and D. Influenza A and B viruses infect humans, causing seasonal influenza epidemics and periodic pandemics due to antigenic drift and antigenic shift, respectively. Influenza A viruses also infect a variety of non-human species (including birds, pigs, horses, cats, dogs, seals and whales), and wild aquatic birds (such as wild ducks, geese, swans, gulls and terns) are their natural

Keywords: Influenza; Anti-influenza agents; Antivirals; RNA interference; siRNA Abbreviations: AGO2, argonaute 2; DNA, deoxyribonucleic acid; dsRNA, doublestranded RNA; HA or H, hemagglutinin; HEF, hemagglutinin-esterase-fusion; HPAI, highly pathogenic avian influenza; M, matrix; M1, matrix 1; M2, matrix 2; miRNA, microRNA; mRNA, messenger RNA; NA or N, neuraminidase; NCR, noncoding regions; NEP, nuclear export protein; NP, nucleoprotein; NS, non-structural; NS1, non-structural 1; NS2, non-structural 2; PA, polymerase acid; PB1, polymerase base 1; PB1-F2, polymerase base 1-F2; PB2, polymerase base 2; piRNA, PIWI-interacting RNA; PTGS, post-transcriptional gene silencing; RdRP, RNA-dependent RNA polymerase; RISC, RNA-induced silencing complex; RNA, ribonucleic acid; RNAi, RNA interference; RSV, respiratory syncytial virus; SARS, severe acute respiratory syndrome; shRNA, short hairpin RNA; siRNA(s), small interfering ribonucleic acid(s); ssRNA, single-stranded RNA; TCAD, triple combination antiviral drug; vRNP, viral ribonucleoprotein.

Received: October 14, 2016; Revised: December 23, 2016; Accepted: January 17, 2017

*Correspondence to: Oluwagbenga A. Adeola, Virology Unit, Department of Medical Microbiology and Parasitology, College of Medicine, Bingham University, Nigeria. Tel: +234-7061275548, E-mail: phamo2@yahoo.co.nz

How to cite this article: Adeola OA. Treatment of Influenza: Prospects of Post-Transcriptional Gene Silencing Through Synthetic siRNAs. Explor Res Hypothesis Med 2017;2(1):1-11. doi: 10.14218/erhm.2016.00013. hosts. ${ }^{3,4}$ Influenza type $\mathrm{C}$ infections generally cause a mild respiratory illness and are not usually associated with epidemics. Influenza D viruses primarily infect cattle and are not known to infect or cause influenza in humans. ${ }^{5}$ The influenza A viruses are classified into 18 hemagglutinin (HA) and 11 neuraminidase (NA) subtypes. Influenza B viruses have no subtypes, but are described by lineages and strains. Common influenza A subtypes in humans include $\mathrm{H} 1, \mathrm{H} 2, \mathrm{H} 3$, N1 and N2, while the less common strains include H5, $\mathrm{H} 7$ and $\mathrm{H} 9$. The currently circulating influenza $\mathrm{B}$ viruses belong to one of two lineages: B/Yamagata and B/Victoria. ${ }^{5}$

Influenza A and B viruses each contain eight genomic segments of negative-sense single-stranded RNA (ssRNA), while the genome of influenza $\mathrm{C}$ virus has seven segments. Each ssRNA segment encodes at least one protein. ${ }^{6}$ For almost three decades after the genome of influenza A viruses was first mapped, the genome of influenza A viruses was thought to encode 10 proteins. However, new findings have revealed that this eight-segment genome could encode up to 18 proteins, and more proteins could be discovered in the future. ${ }^{7}$ The 10 proteins initially recognized are polymerase basic protein 2 (PB2), polymerase basic protein 1 (PB1), polymerase acidic protein (PA), HA, nucleocapsid protein or nucleoprotein (NP) and NA, encoded by segments 1, 2, 3, 4, 5 and 6, respectively. They also include matrix 1 (M1) and matrix 2 (M2) proteins (from segment 7) and non-structural protein 1 (NS1) and non-structural protein 2 (NS2; also known as nuclear export protein, NEP) (from segment 8$){ }^{6-8}$ The first accessory protein, PB1-F2, translated from the fourth AUG codon in an alternative reading frame of PB1, was identified in 2001. It is a small, 87-residue protein that enhances apoptosis in immune cells and is thought to aid viral transmission. Other novel proteins encoded by the genome of influenza A viruses include PB1-N40 (which is translated from the fifth AUG 


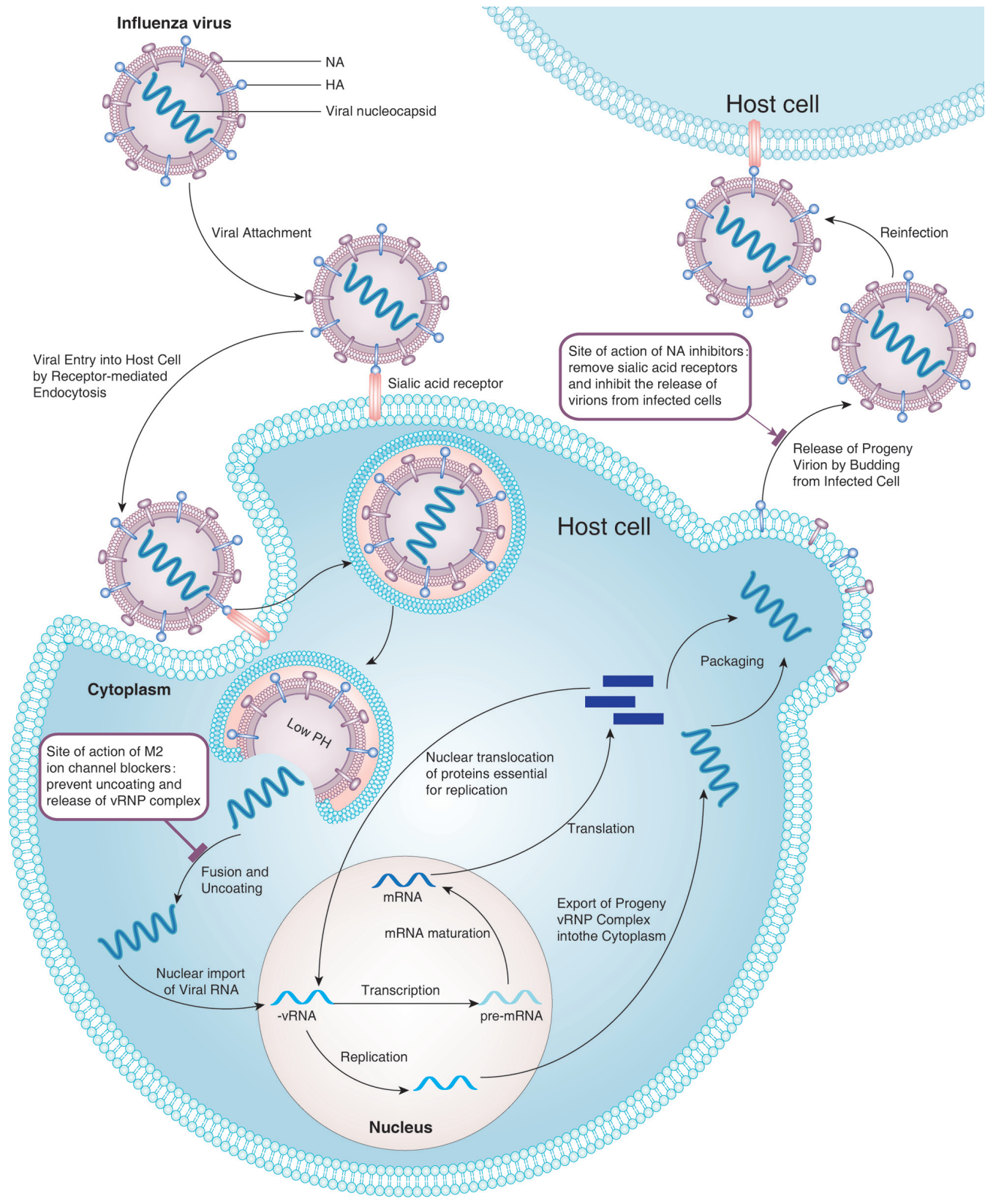

Fig. 1. Schematic diagram of the life cycle of influenza viruses showing the sites of action of the two main groups of currently available traditional anti-influenza drugs. Green arrows depict important steps in the life cycle of influenza viruses; red arrows show the sites of action of M2 ion channel blockers and NA inhibitors; black arrows represent the sequence of events during transcription, translation, and genomic replication: after nuclear import of vRNA, transcription by viral RNA-dependent RNA polymerase produces initial mRNA transcripts (pre-mRNA) which then undergo a maturation phase (including splicing) before export into the cytoplasm where translation occurs to produce new viral proteins; some newly produced viral proteins (such as polymerases and NEP) are translocated back into the nucleus for initiation of genome replication and subsequent export of progeny vRNPs.

codon that is in-frame with the PB1 start codon), PA-X (a ribosomal frame-shifting derivative of segment 3), PA-N155, PA-N182, M42 and NS3. On the other hand, the eight-segment genome of influenza B virus encodes 11 proteins, while the seven-segment genome of influenza $\mathrm{C}$ virus encodes 9 proteins. ${ }^{7-10}$

Proteins encoded by influenza $\mathrm{B}$ viruses (and their encoding segments) are PB1 (segment 1), PB2 (segment 2), PA (segment 3), HA (segment 4), NP (segment 5), NA and NB (as overlapping open reading frames on segment 6), M1 (segment 7), BM2 (segment 7, through a translational stop-start mechanism), and NS1 and NS2 (segment 8). The BM2 protein is important for incorporation of the viral ribonucleoprotein (vRNP) complex into virions during virion assembly, and is therefore crucial for generation of infectious progeny influenza $\mathrm{B}$ viruses. Those encoded by influenza $\mathrm{C}$ virus (and their encoding segments) are PB2 (segment 1), PB1 (segment 2), P3 (segment 3), hemagglutinin-esterase-fusion (HEF) (segment 4), NP (segment 5), M1 and CM2 (segment 6), and NS1 and NS2 (segment 7). Functions of the HEF protein include receptor bind- 
ing, esterase activity and membrane fusion, while CM2 may have a role in genome packaging and uncoating during replication of influenza $\mathrm{C}$ viruses. ${ }^{\mathbf{8} 9}$ Treatment of seasonal influenza and prevention of pandemic influenza have become major issues in recent years, due to a high rate of evolution of influenza viruses and ensuing resistance to existing anti-influenza drugs and vaccines. Novel and efficient treatment strategies are therefore urgently needed. This review discusses the prospects and use of small interfering RNAs (siRNAs) as treatment of influenza. Challenges associated with siRNA interference are also highlighted.

Life cycle of influenza virus and currently available options for influenza therapy

Influenza viruses replicate in the host cell nucleus, unlike most RNA viruses. As shown in Figure 1, their replication cycle can be divided into the following stages: attachment to host cell receptors; viral entry by endocytosis; release of viral nucleocapsid into the cytoplasm by fusion of viral and endosomal membranes (mediated by low $\mathrm{pH}$ within the endosome); transport of viral negativesense RNA into the nucleus; primary mRNA transcription by viral polymerases; transport of primary mRNA transcripts into the cytoplasm and subsequent translation to produce new polymerase, NP, NS1 and NS2 proteins; transport of newly produced viral proteins back into the nucleus to initiate genomic replication and secondary mRNA transcription; assembly of viral ribonucleoprotein complex in the nucleus and subsequent export to the cytoplasm; and release of new virions from infected cells through budding from the apical side of cytoplasmic membrane. The release of progeny virions from infected cells is achieved through the activity of NA proteins, which cleave and remove sialic acid receptors from cellular glycoproteins and glycolipids. ${ }^{10-12}$

Two major classes of drugs have been used for treatment of influenza virus infection. These are the M2 ion channel blockers and inhibitors of NA (Fig. 1).

\section{M2 ion channel blockers}

The M2 protein is an ion (proton) channel that allows proton translocation through the virion envelope, thereby leading to acidification of the viral core and subsequent dissociation and release of the vRNP complex in the host cell cytoplasm. This step is vital for the function of viral RNA-dependent RNA polymerase (RdRP) and expression of viral genes. ${ }^{13}$ M2 ion channel blockers, which include amantadine and rimantadine (adamantanes), were the first generation of influenza antiviral agents. They inhibit this important function of the M2 protein, but due to high resistance, their use is now largely discontinued and replaced by NA inhibitors. ${ }^{14-16}$ In addition, their efficacy was restricted to influenza A viruses only, since influenza B viruses do not possess the M2 protein. ${ }^{17}$

\section{Inhibitors of $\mathrm{N} A$}

As stated earlier, the NA protein cleaves and removes sialic acid receptors from cellular glycoproteins and glycolipids, thereby releasing new virions from infected cells. ${ }^{6,10}$ Thus, inhibitors of NA interrupt an established infection in its late stages by inhibiting the release of virions from infected cells. This leads to accumulation of virions at the apical cell surface, consequent inhibition of viral penetration of mucous secretions, and prevention of spread to other cells. ${ }^{15,18}$ The three currently approved anti-influenza drugs in the U.S., recommended by the Centers for Disease Control and Prevention (CDC), are all inhibitors of NA. They are oseltamivir (oral), zanamivir (inhalational), and peramivir (intravenous/intramuscular). ${ }^{19}$

NA inhibitors are active against most influenza A and B viruses and elicit fewer side effects, but development of resistance to oseltamivir and zanamivir by different influenza viruses has been reported. ${ }^{20}$ For instance, sporadic oseltamivir-resistant 2009 H1N1 virus infections have been reported, occasionally with rare episodes of limited transmission, and more of such cases can be expected. ${ }^{20-23}$ In fact, oseltamivir-resistant 2009 H1N1 virus strains have been reported to emerge within 48 hours after initiation of treatment. ${ }^{24}$ In addition, transmission of oseltamivir-resistant influenza B virus strains or $2009 \mathrm{H} 1 \mathrm{~N} 1$ virus strains acquired from persons treated with oseltamivir rarely occurs but has been documented, and clinical isolates with reduced susceptibility to zanamivir have been obtained occasionally from immunocompromised children on prolonged therapy. ${ }^{21,25-27}$ Development of resistance to zanamivir or oseltamivir has also been identified during treatment of seasonal influenza. ${ }^{23,28}$

\section{Inhibitors of viral RNA synthesis}

Although ribavirin, a nucleoside analogue, shows activity against influenza viruses, it is less active against the influenza viruses than M2 channel blockers or NA inhibitors. ${ }^{29,30}$ Several reports also posited that significant safety issues, such as the risk of hemolytic anemia and of teratogenicity, present additional challenges to address if ribavirin is to be used for the treatment of influenza. ${ }^{30,31}$

\section{Combination therapy with amantadine, oseltamivir and ribavi- rin}

A triple combination antiviral drug (TCAD) regimen composed of amantadine, ribavirin and oseltamivir produced considerable antiviral effect against drug-resistant seasonal and 2009 H1N1 influenza viruses in vitro. ${ }^{32,33}$ However, in a retrospective study on critically-ill A/H1N1pdm09 influenza patients, those who were treated with TCAD showed little additional toxicity and an insignificant trend towards lower mortality than those who were treated with only oseltamivir. ${ }^{34}$ Thus, results from in vitro assessment of TCAD do not necessarily imply good in vivo antiviral activity, and safety of TCAD has not been fully investigated.

Problems associated with influenza vaccination and chemotherapy

Several factors contribute to the complexity associated with prevention and treatment of influenza. These include the following: high rate of mutation; genetic reassortment; and high risk human populations.

\section{High rate of mutation}

RNA viruses, including influenza viruses, generally mutate faster (typically at or near the error threshold for maintaining the integrity of genetic information) than DNA viruses, due to lack of exonuclease proofreading activity of the virus-encoded RdRP. Mutation rates for RNA viruses were found to range from $10^{-6}$ to $10^{-4}$ sub- 
stitutions per nucleotide per cell infection $(\mathrm{s} / \mathrm{n} / \mathrm{c})$ for RNA viruses, in comparison to $10^{-8}$ to $10^{-6} \mathrm{~s} / \mathrm{n} / \mathrm{c}$ found for DNA viruses. ${ }^{35}$ This high mutation rate contributes to the highly adaptive capacity of RNA viruses. ${ }^{36,37}$ High mutation rates may also increase the rate at which RNA viruses, including influenza viruses, adapt to a new host, acquire a new route of transmission, escape from host immune surveillance, or become resistant to existing vaccines and antiviral drugs. ${ }^{38,39}$ For instance, resistance to oseltamivir and zanamivir has been attributed to mutations in the NA and HA genes. ${ }^{40-43}$

In addition, although most viral mutations are either lethal or highly detrimental to the virus, influenza viruses have been found to have considerable mutational robustness, with mutations in genomic segments 4 (HA) and 6 (NA) appearing to be less arduously accommodated than elsewhere in the genome. ${ }^{37,39}$ This relative ease of accumulation of mutations in the HA and NA genes may allow the virus to escape from the host's specific immunity and sensitivity to available drugs. ${ }^{44}$ A typical example is $\mathrm{H} 275 \mathrm{Y}$ mutation in the NA gene of influenza viruses. Because oseltamivir loses its ability to bind to and inhibit the function of the mutant's NA proteins, such mutants become resistant to oseltamivir and they spread more easily to other uninfected cells. ${ }^{45}$

\section{Genetic reassortment}

Genetic reassortment or 'genetic mixing' usually occurs between genomic segments of co-infecting influenza viruses in a permissible host. The domestic pig, which has been termed a 'mixing vessel' for influenza viruses, can be co-infected with avian and human influenza viruses, leading to genetic reassortment and generation of novel strains of influenza viruses through antigenic shift. Reassortment, which typically occurs within a genus and not between different genera, can produce new genome constellations. ${ }^{13}$, 46-48 Preventive strategies targeting the human-swine interface are therefore important for prevention of future pandemics. ${ }^{49}$

\section{Human infection by 'variant' influenza viruses}

Swine influenza viruses do not normally infect humans. However, sporadic human infections with typical swine influenza viruses have occurred, and these are called 'variant' viruses (denoted by adding ' $v$ ' to the end of the virus subtype designation). Human infections with variant H1N1 (H1N1v), variant H3N2 (H3N2v), and variant $\mathrm{H} 1 \mathrm{~N} 2(\mathrm{H} 1 \mathrm{~N} 2 \mathrm{v})$ swine influenza $\mathrm{A}$ viruses have been detected in the United States. Most commonly, human infections with these variant viruses have occurred in people with significant exposure to infected pigs. This category includes live-pig handlers at abattoirs and slaughter slabs, pig farm workers, and children who have close contacts with pigs at fairs..$^{50,51}$ While the efficacy of currently available anti-influenza drugs for treatment of these variant viruses is akin to that of seasonal influenza viruses, some variant viruses, specifically currently circulating $\mathrm{H} 3 \mathrm{~N} 2 \mathrm{v}$ viruses, are known to acquire the $\mathrm{M}$ gene of the 2009 pandemic H1N1 influenza virus. This makes $\mathrm{H} 3 \mathrm{~N} 2 \mathrm{v}$ infect humans more easily than is typical for other swine influenza viruses. ${ }^{52}$

\section{Need for commencement of influenza treatment at the onset of disease}

Ideally, influenza drugs should be administered early during the course of the disease, optimally within 48 hours of onset of symptoms. ${ }^{2,16}$ This may be difficult to achieve, especially in developing countries, either due to unavailability or inaccessibility of drugs, or inability of affected persons to correctly attribute the non-specific clinical signs noticeable at this early stage to influenza. ${ }^{49}$

\section{High-risk human populations}

Influenza is typically mild, usually with recovery occurring in less than two weeks. However, complications, such as pneumonia, bronchitis, sinus infections and ear infections, may occur in some high-risk groups. Such complications could result in hospitalization and sometimes death. Vulnerable groups include: children younger than 5 (especially those below 2 ) years-old; elderly people who are at least 65 years-old; women during pregnancy and up to two weeks postpartum; residents of nursing homes and other longterm hospices; persons with immunodeficiency disorders, such as acquired immune deficiency syndrome; and American Indians and Alaskan natives (who have been found to have higher likelihood for hospitalization due to influenza than the general U.S. population). ${ }^{53,54}$ This vulnerable population, therefore, has greater need for treatment because while they constitute $<20 \%$ of the overall population, the highest mortality rates during seasonal influenza are usually associated with them. However, their low tolerance for anti-influenza drugs puts a limit on their use of these drugs. ${ }^{43}$ This could become a serious problem if not adequately managed.

\section{siRNAs: Historical Highlights}

The discovery of RNA interference (RNAi) in the 1990s revolutionized our understanding of gene regulation. RNAi is a naturally occurring endogenous post-transcriptional cellular mechanism that regulates RNA expression. ${ }^{\mathbf{5 5 , 5 6}}$ In the early 1990 s, researchers observed that RNA inhibited protein expression in plants and fungi. ${ }^{57}$ This phenomenon, which was not well understood at the time, was known as post-transcriptional gene silencing (PTGS), quelling, and co-suppression. ${ }^{\mathbf{5 8 , 5 9}}$ In 1998, Andrew Fire and Craig C. Mello observed, in the nematode Caenorhabditis elegans, that double-stranded RNA (dsRNA) was the source of sequence-specific inhibition of protein expression, which they referred to as $R N A$ interference. ${ }^{60}$ They later won the Nobel Prize in Physiology or Medicine, in 2006, for their discovery of RNA interference. Key events in the history of RNAi are summarized in Table $1 . .^{58-70}$

siRNA (also referred to as short interfering RNA or silencing RNA) is a class of 18-26 (typically 21-25) base-pair long dsRNA, which function within the RNAi pathway by interfering with the expression of specific genes with complementary nucleotide sequences by degrading mRNA after transcription, thereby resulting in no translation. ${ }^{56,71}$ The earliest suggestion that a synthetic siRNA can act as an antiviral came in 2001 when the growth of respiratory syncytial virus (RSV) was inhibited by synthetic, 21 nucleotide-long, dsRNA molecules that degraded the essential viral mRNAs, thereby preventing specific viral protein formation. ${ }^{65}$ The first report in which the siRNA-based approach was successfully used against influenza was made in $2003 .{ }^{66}$

\section{Mechanism of RNAi}

Several pathways exist for RNAi. These are microRNA (miRNA), siRNA, short hairpin RNA (shRNA), and the germ line-specific PIWI-interacting RNA (piRNA) pathways. ${ }^{57,72-75}$ Initially, silencing of specific genes in mammalian cells was difficult because of 
Table 1. Important landmarks in the history of RNA interference

\begin{tabular}{|c|c|c|}
\hline Key Events & Timeline & Reference \\
\hline $\begin{array}{l}\text { Co-suppression of endogenous chalcone synthase (CHS) gene in petunias } \\
\text { plant }\end{array}$ & 1990 & Napoli and Jorgensen ${ }^{58}$ \\
\hline Quelling of endogenous gene in the fungi Neurospora crassa & 1992 & Romano and Macino ${ }^{59}$ \\
\hline $\begin{array}{l}\text { First documentation of RNAi in animals (Caenorhabditis elegans) using } \\
\text { antisense RNA }\end{array}$ & 1995 & Guo and Kemphues ${ }^{61}$ \\
\hline $\begin{array}{l}\text { Explication of dsRNA as the basis of sequence-specific inhibition of } \\
\text { protein expression; Coinage of the term RNA interference }\end{array}$ & 1998 & Fire et al. ${ }^{60}$ \\
\hline Discovery of siRNAs as mediators of RNAi through gene 'knock down' & 2000 & Hammond et al.; Zamore et al. ${ }^{62,63}$ \\
\hline Discovery of RNA-induced silencing complex (RISC) & 2001 & Bernstein et al. ${ }^{64}$ \\
\hline $\begin{array}{l}\text { Elucidation of the Dicer as a requisite for the initiator phase of RNAi } \\
\text { pathway }\end{array}$ & 2001 & Bernstein et al. ${ }^{64}$ \\
\hline $\begin{array}{l}\text { Earliest indication of use of synthetic siRNA as antiviral agents } \\
\text { (inhibition of growth of RSV) }\end{array}$ & 2001 & Bitko and Barik ${ }^{65}$ \\
\hline First report on use of siRNA as anti-influenza agent & 2003 & Ge et al. ${ }^{66}$ \\
\hline Discovery of the Slicer activity of AGO2 & 2004 & Song et al.; Martinez and Tuschl; Liu et al. ${ }^{67-69}$ \\
\hline $\begin{array}{l}\text { Nobel Prize in Physiology or Medicine to Andrew Fire and Craig C. } \\
\text { Mello for discovery of RNAi }\end{array}$ & 2006 & Nobel Media AB $2014^{70}$ \\
\hline
\end{tabular}

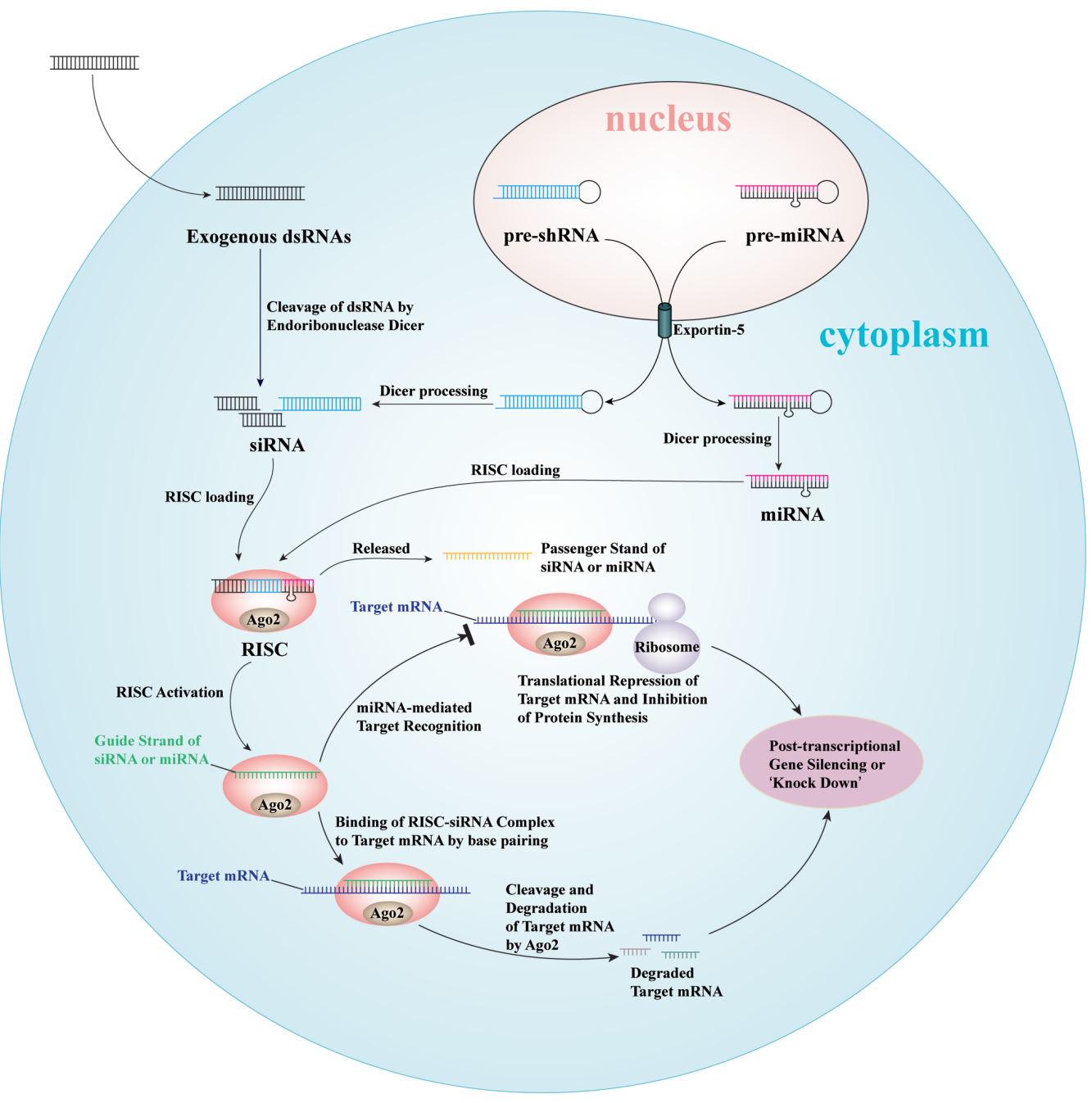

Fig. 2. Pathways of RNA interference. 
the induction of the interferon response by dsRNAs of $\geq 30$ nucleotides. ${ }^{75-77}$ However, the discovery that siRNA actually mediates RNAi through PTGS or 'knock-down' increased the level of interest in their use as antiviral agents. ${ }^{56,63,78,79}$

Two key differences between miRNA and siRNA are their cellular origins and the nature of the dsRNA precursors of each. Whereas miRNAs are derived from the genome, siRNAs may be endogenous or arise via viral infection or other exogenous sources. ${ }^{80}$ In addition, siRNA duplexes feature perfect base-pairing, whereas miRNA helices contain mismatches and more extended terminal loops. A shRNA is a synthetic RNA molecule with a tight hairpin turn that could be used to silence target gene expression by RNAi. Although shRNA is an advantageous mediator of RNAi in that it has a relatively low rate of degradation and turnover, expression of shRNA in cells is usually accomplished by delivery of plasmids or through viral or bacterial vectors. This requirement for an expression vector could pose safety concerns. Moreover, subsequent to their expression in the nucleus, the precursors of shRNAs are processed by Drosha and then exported by Exportin-5 to the cytoplasm, where the loop sequence of the shRNA is removed by Dicer. From this stage, shRNAs are processed in the same manner as siRNAs. ${ }^{81-83}$ Thus, although several pathways exist for RNAi, siRNA and miRNA are fundamental to RNAi. Figure 2 shows the pathways of RNAi by siRNA and miRNA.

RNAi is therefore initiated by exposing cells to siRNA or dsRNAs (via transfection or endogenous expression). ${ }^{75}$ The naturally occurring dsRNAs are processed by the dsRNA-specific endonuclease Dicer-RDE-1, which cleaves long dsRNA into smaller double-stranded duplexes (usually 21-23 nucleotides in length) of siRNA. ${ }^{84}$ The siRNAs then form a complex with the RNA-induced silencing complex (RISC), and subsequently guide the RISC to cleave target mRNAs that share sequence identity with the siRNA, as follows. ${ }^{64,75,76}$ After incorporation into the RIC complex, one strand of the siRNA duplex (referred to as the passenger strand) is cleaved by an endonuclease of the RISC, the Argonaute 2 (AGO2), leading to activation of the RISC. This strand is released. ${ }^{85,86}$ The other strand (referred to as the guide strand) then links the activated RISC (now with a single-strand siRNA) complex to the target mRNA by base pairing. Target mRNA is then cleaved and degraded by AGO2, thereby preventing translation and synthesis of associated proteins. This effectively results in a silenced gene (PTGS or 'knock-down'). ${ }^{85,86}$ Thus, all of these processing pathways (of miRNA, siRNA, shRNA and piRNA) converge at the point of assemblage of the RNA into the RISC and they subsequently proceed through a common mode of action. ${ }^{74}$ Introduction of siRNA into cells, therefore, leads to down-regulation or 'knock-down' of target genes without triggering interferon responses. ${ }^{84}$

\section{Advantages of siRNAs over traditional anti-influenza drugs}

RNAi of specific influenza genes by siRNAs has several advantages over organic anti-influenza drugs. These include the ease of synthesis, superior efficiency of production, and less susceptibility to resistance. These advantages are highlighted below:

\section{Ease of synthesis and better efficiency of production}

One of the key benefits of siRNA-based therapeutics during pandemic influenza is that the basic requirement for design of specific siRNAs is knowledge of the gene sequence of the pandemic strain. Once the sequence has been elucidated, appropriate siRNAs could be synthesized at relatively low cost within a short period. ${ }^{87}$
Moreover, because the design algorithms for siRNA-based agents are nearly identical for any pathogen, synthetic and mass production processes for all siRNAs are the same, and their efficiency of production is better. ${ }^{43,88}$

\section{Less susceptibility to resistance}

Several studies have reported that siRNA-based drugs are less susceptible to development of resistance by influenza viruses. ${ }^{43,79,89}$ For instance, while Inoue et al. reported that oseltamivir-resistant 2009 pandemic H1N1 virus strains have been shown to emerge within 48 hours after initiation of treatment, Sui and colleagues reported that when $\mathrm{H} 1 \mathrm{~N} 1$ and highly pathogenic avian influenza (HPAI) H5N1 viruses were cultured in shM2-MDCK cells (MDCK cells expressing shRNAs M2-950), no mutation appeared in the region targeting siM2 (siRNA targeting influenza M2 gene) even after 40 passages of the cultures. ${ }^{24,79}$ Moreover, even if resistance to a particular siRNA targeting one influenza gene segment develops, another siRNA, targeting another genomic segment could be used. ${ }^{43}$

\section{Broad anti-influenza spectrum and long-term inhibitory effect}

In a recent study, the antisense oligonucleotide designed against the noncoding regions (NCR) of influenza virus was able to inhibit the expression of the viral genome of several influenza virus strains. The presence of antisense oligonucleotide showed reduction of virus multiplication and pathogenesis both in vitro and in vivo. This implied that a single antisense oligonucleotide could provide protection against more than one strain of influenza A virus. ${ }^{90}$ Another in vivo study carried out by Tompkins et al. revealed that influenza-specific siRNA treatments directed against the NP and PA genes were broadly effective and protected animals against lethal challenge with highly pathogenic avian influenza A viruses of the $\mathrm{H} 5$ and $\mathrm{H} 7$ subtypes. ${ }^{91} \mathrm{Ge}$ and co-workers also reported that siRNAs targeting the NP and PA gene segments (NP-1496 and PA-2087, respectively) provided a broadly effective inhibition of H1N1 influenza virus. ${ }^{66}$ This is because these siRNAs did not only inhibit the accumulations of specific NP or PA mRNAs, but also inhibited the accumulations of mRNAs for M, NS1, PB1, PB2 and PA or NP genes. This broadly effective inhibition was thought to be as a result of interferon response or activation of an RNA degradation pathway (such as phosphorylated protein kinase R, PKR) used by some siRNAs. ${ }^{92,93}$

Another benefit of many anti-influenza siRNAs is their longterm inhibitory effect. In a recent report, Behera and co-workers observed, using three siRNAs designed against the PB2 and NP gene segments of HPAI H5N1 (identified as PB2-2235, PB2-479 and NP-865), 8- to 16 -fold reduction in virus HA titers, $68-80 \%$ reduction in viral plaque counts, and $87-94 \%$ reduction in RNA copy number for 48 hours (maximum observation period) after challenge with 100 TCID50 of virus. ${ }^{94}$ Sui et al also reported that inhibition of propagation of H1N1 and HPAI H5N1 viruses in stable cell lines by siRNAs targeting the M2 gene was sustained for more than 50 hours post-infection. ${ }^{79}$ Similar results were obtained by Ge et al. in MDCK cells using siRNA targeting the NP gene (NP-1496). ${ }^{66}$

\section{Possibility of intranasal administration}

It has been shown that intranasal delivery contributes to the efficacy of siRNAs more than intravenous delivery. ${ }^{66,91}$ Intranasally 
administered siRNAs have been developed, and they are reported to show prompt delivery to the lungs, leading to significant protection of animals. ${ }^{95}$ They also have the added advantage of being effective when used therapeutically and prophylactically. ${ }^{87}$ For instance, Bitko et al. reported that siRNAs administered intranasally, with or without transfection reagents, in the mouse model specifically inhibited respiratory viruses. ${ }^{95}$ These authors also observed that the degree of protection in mice was comparable to the antiviral activity of the siRNA in cell culture. Zhang and co-workers also reported that intranasal treatment of mice with siNS1 (siRNA targeting the NS1 gene) nanoparticles, either before or after viral infection, showed significant decrease in pulmonary virus titers, inflammation and airway reactivity, compared to controls. ${ }^{96}$ Moreover, an siRNA-based inhaler has been successfully developed against RSV and could also work for influenza. ${ }^{87}$

\section{Possibility of use as anti-influenza agent in human and animals}

Some previous reports showed that siRNA targeting influenza M2 gene potently inhibited viral replication, and was effective, not only for H1N1 virus but also for H5N1 highly pathogenic avian influenza virus. ${ }^{79}$ Another study also revealed that siRNAs could effectively reduce the NS1 gene expression of the H5N1 avian influenza virus. ${ }^{97}$ Sirnaomics have developed a siRNA-based therapeutic agent against influenza virus, known as STP702. STP702 has been shown to be effective against H5N1 (avian flu) and H1N1 (swine flu). In this application, several siRNAs targeting conserved regions of the influenza virus genome have been combined to form a cocktail that has a multitargeted approach. ${ }^{88}$

Furthermore, Rajput et al. reported that when NS1-specific siRNA was administered in experimental mice, a $92 \%$ reduction was observed in the levels of NS1 gene expression in the lungs. ${ }^{98}$ Significant decrease in cytokine levels and virus titers in the lungs were also observed. Zhou et al. also designed three siRNAs against the NP, PB1 and PA proteins of $\mathrm{H} 5 \mathrm{~N} 1$ avian influenza virus. ${ }^{99}$ Their findings revealed that newly synthesized nucleocapsid, PA and $\mathrm{PB} 1$ proteins are required for avian influenza virus transcription and replication and could serve as a basis for the development of siRNAs as prophylaxis and therapy for avian influenza infection in birds and humans.

\section{Concise appraisal of some synthetic anti-influenza siRNAs}

In mammalian cells, the requirement for Dicer-RDE-1-mediated processing of long dsRNA RNAi could be bypassed by introducing synthetic 21 -nt siRNA duplexes into the cells. ${ }^{100}$ While such 21-nt siRNAs are too short to induce an interferon response in mammalian cells, they retain the ability to confer transient interference of gene expression in a sequence-specific manner. ${ }^{100,101}$ These exogenous 21 -nt siRNAs therefore represent a previously unrecognized class of molecules that may have significant medical applications. ${ }^{66}$ Their processing subsequently follows the same pathways as naturally occurring siRNAs. This implies that within the cytoplasm, the duplexes become separated by the RISC. ${ }^{102}$ Various siRNAs targeting different influenza gene segments have been produced. For instance, siRNA designed against the M2 (siM2) gene conferred long-term effective inhibition of influenza A virus replication. In addition, siRNA-resistant mutants did not appear in the targeted sequence, even after culturing the virus for 40 passages. ${ }^{79}$ Among the three siRNA expression plasmids designed by Zhou et al. against H5N1 avian influenza, siRNA specific for nu- cleocapsid protein was found to have a specific effect in inhibiting the accumulation of RNAs in infected cells because of a critical requirement for newly synthesized nucleocapsid proteins in avian influenza viral RNA transcription and replication. ${ }^{99}$ Tompkins et $a l$. also produced broadly effective anti-influenza siRNAs directed against the NP and PA genes. ${ }^{91}$ These siRNAs protected animals against lethal challenge with highly pathogenic avian influenza $\mathrm{A}$ viruses of the $\mathrm{H} 5$ and $\mathrm{H} 7$ subtypes.

\section{Challenges associated with clinical use of anti-influenza siRNA}

Clinical use of siRNA-based drugs has been limited as a result of several challenges. Questions abound on the pharmacokinetics, modalities for targeted delivery, and possibility of off-target or inadvertent effects of siRNA-based drugs. ${ }^{102}$ A major limitation to utilizing siRNAs for treatment in humans is the development of efficient delivery systems. ${ }^{103}$ For instance, although inhaled delivery of naked, modified and complexed siRNAs has shown efficacy for respiratory targets, such delivery of naked siRNAs also has its low points. Such limitations include the fact that early in vivo efforts at topical delivery of siRNA revealed that topical formulations could have cytotoxic effects. ${ }^{102}$ More studies on pharmacokinetics and targeted delivery of specific anti-influenza siRNAs in appropriate animal models would be crucial in overcoming these limitations. In this regard, the ferret, which has been described as a model organism for influenza A virus infection studies, would be an appropriate candidate. ${ }^{104,105}$ Premised on a study which reported that combining different delivery strategies enhanced the efficacy of synthetic anti-influenza siRNAs, the possibility of combining intranasal delivery and another delivery strategy, such as hydrodynamic intravenous delivery, could also be explored. ${ }^{91}$ Such combination could be assessed starting with intranasal delivery followed by another delivery method, or they could be tested concurrently.

Off-target effect occurs when a siRNA, subsequent to processing by the RISC, knocks down unintended targets. In other words, it connotes a siRNA-mediated silencing of unintended mRNA transcripts. ${ }^{106}$ First described in 2003, siRNA-mediated off-target effects, which were initially thought to be inconsequential, are now known to produce false positives in siRNA screens, induce measurable phenotypes, and may cause undesired side effects during clinical trials. ${ }^{106-111}$ It has been shown that off-target effects occur as a result of complementarity between the seed region of the siRNA (nucleotide positions 2-7) and sequences in the 3'-UTR of the off-targeted gene. ${ }^{106,109,112,113}$ Off-target effects have also been reported to be concentration-dependent. This implies that that the higher the concentration of the siRNA, the greater the likelihood of occurrence of off-target effects. ${ }^{106,107,114}$

In recent years, approaches that have been used to reduce siRNA-mediated off-target effects include fine-tuning of siRNA concentration, chemical and structural modification of siRNA, and application of more extensive and painstaking bioinformatics for improvement of siRNA design. To start with, as noted earlier, offtarget effects are concentration-dependent, and are therefore expected to be reduced as the concentration of the siRNA is reduced. To ensure that depletion of target gene knock-down does not occur along with reduction of off-target effects, studies have revealed that using a pool consisting of highly functional multiple siRNAs in place of individual siRNA could ensure production of strong ontarget gene knock-down with negligible off-target effects. ${ }^{106,108,111}$

Off-target effects could also be reduced through chemical and structural modification of siRNA. Chemical modifications could 
involve either the sense strand, the antisense strand, or both strands. For instance, Jackson and co-workers reported that positionspecific chemical modification (2'-O-methyl ribosyl substitution at position 2 in the guide strand) reduced gene silencing of most off-target transcripts that had complementarity to the seed region of the siRNA guide strand. ${ }^{115}$ Dua et al. also developed a modified backbone structure for siRNA with a single nucleotide bulge placed in the antisense strand (termed 'bulge-siRNA'), and found that presence of a bulge at position 2 of the antisense strand enabled the siRNA to better discriminate between perfectly matched and mismatched targets, with no loss in on-target gene silencing. ${ }^{116}$

Bioinformatics could also be a veritable tool for minimizing siRNA-mediated off-target effects. As previously noted, off-target effects occur as a result of complementarity between the seed region of the siRNA and sequences in the 3'-UTR of the off-targeted gene. Thus, bioinformatics tools could be used, during siRNA design, to minimize off-target effects. ${ }^{117-119}$ For instance, Yilmazel and colleagues have designed a tool for prediction of off-target effects in large-scale RNAi screen data via seed region analysis (named 'Online GESS'). ${ }^{120}$

Furthermore, high output screening of siRNAs is challenging due to inconstancy of cell culture requirements and transfection efficiency. To mitigate this challenge, multiple siRNAs or pools (3-6 siRNAs) could be used to target a specific gene. The pools of siRNAs can be initially screened for efficacy, before analyzing each siRNA in the pool. In spite of the possibility of competition between poorly performing and efficient siRNAs in each pool, and the resultant lowering of the efficacy of the pooled siRNAs, the relatively higher output and reduced cost of using siRNA pools would outweigh this potential for false negatives. ${ }^{102}$

\section{Future directions}

The acceptance of siRNA-based anti-influenza treatments is expected to increase significantly in the next few years. This is because, coupled with the enormous advantages of siRNA over currently approved anti-influenza therapeutic approaches, novel strategies for efficient delivery of siRNA-based drugs are expected to evolve. Most recent reports have shown that nanoparticles could serve efficiently for siRNA delivery. Such nanoparticles include acoustically propelled nanomotors for intracellular siRNA delivery and lipid-based nanoparticles used as carriers for RNAi against viral infections. ${ }^{121,122}$ Attempts should therefore be made, using appropriate animal models such as the ferret, to exploit the use of nanoparticles for intracellular delivery of anti-influenza siRNAbased drugs. Furthermore, to significantly minimize siRNA-mediated off-target effects while ensuring effective silencing of target influenza virus genes, effort should be put into using pools consisting of moderate and effective concentrations of highly functional multiple siRNA targeting different segments of the genome of influenza viruses in place of high concentrations of a siRNA targeting only one gene segment.

\section{Conclusion}

An efficient siRNA-based treatment of influenza must possess a number of attributes, including negligible toxicity and immune reaction, adequate and specific tissue delivery to the upper and lower respiratory tracts, pharmacokinetic stability, long-term inhibitory effect, broad anti-influenza spectrum, and high resistance to development of siRNA-resistant mutants in vivo. While modali- ties for targeted delivery and possibility of unintended effects of siRNA-based drugs constitute crucial challenges in need of urgent solutions, the use of synthetic siRNAs for treatment of influenza virus infections has considerable advantages over currently available traditional anti-influenza agents. These include relatively higher ease of synthesis and better efficiency of production, and most importantly, the prospect of high resistance to development of siRNA-resistant mutants. The advent of novel target-delivery strategies, such as the use of nanoparticles, and the possibility of reduction of siRNA-mediated off-target effects to negligible levels through optimization of siRNA concentration, chemical and structural modifications, and application of bioinformatics tools for improvement of siRNA design, are expected to significantly increase the acceptance and use of siRNA-based anti-influenza drugs in the next few years.

\section{Conflict of interest}

The author has no conflict of interest related to this publication.

\section{Author contributions}

Literature search (OAA), initial draft (OAA), preparation of figures (OAA), correspondence (OAA), and revision of the paper (OAA).

\section{References}

[1] Kawaoka Y, Cox N, Haller O. Fauquet C, Mayo MA, Maniloff J, et al. Editors. Virus taxonomy: VIIIth report of the international committee on taxonomy of viruses. London: Elsevier Academic Press, 2005;681693.

[2] World Health Organization (WHO) [Internet]. Influenza (Seasonal). Fact sheet No 211, March 2014. c2014 [cited 2016 September 25]. Available from: http://www.who.int/mediacentre/factsheets/fs211/ en/.

[3] Webster RG, Bean WJ, Gorman OT, Chambers TM, Kawaoka Y. Evolution and ecology of influenza A viruses. Microbiol Rev 1992;56(1):152-179.

[4] Kuiken T, Holmes EC, McCauley J, Rimmelzwaan GF, Williams CS, Grenfell BT. Host species barriers to influenza virus infections. Science 2006;312(5772):394-397. doi:10.1126/science.1122818.

[5] Centers for Disease Control and Prevention (CDC) [Internet]. Types of influenza viruses. seasonal influenza (flu)influenza - flu basics: influenza viruses. c2016 [cited 2016 September 24]. Available from: http://www.cdc.gov/flu/about/viruses/types.htm.

[6] Dubois J, Terrier O, Rosa-Calatrava M. Influenza viruses and mRNAS: doing more with less. mBio 2014;5(3):e00070-14. doi:10.1128/ mBio.00070-14.

[7] Vasin AV, Petrova AV, Egorov VV, Plotnikova MA, Klotchenko SA, Karpenko MN, et al. The influenza A virus NS genome segment dis plays lineage-specific patterns in predicted RNA secondary structure. BMC Research Notes 2016;9:279. doi:10.1186/s13104-016-2083-6.

[8] Jackson D, Elderfield RA, Barclay WS. Molecular studies of influenza B virus in the reverse genetics era. J Gen Virol 2011;92:1-17. doi:10.1099/vir.0.026187-0.

[9] Muraki Y, Hongo S. The molecular virology and reverse genetics of influenza C virus. Jpn J Infect Dis 2010;63:157-165.

[10] Samji T. Influenza A: understanding the viral life cycle. Yale J Biol Med 2009;82(4):153-159.

[11] Palese P, Tobita K, Ueda M, Compans RW. Characterization of temperature sensitive influenza virus mutants defective in neuraminidase. Virology 1974;61(2):397-410. doi:10.1016/0042-6822(74)90276-1. 
[12] Ezelle HJ, Hassel BA. Mechanisms of IFN resistance by influenza virus. In: Paul FT, editor. Combating the threat of pandemic influenza: drug discovery approaches. John Wiley and Sons, Inc. 2007.

[13] Palese P, Shaw ML. Orthomyxoviridae: The viruses and their replication. In: Knipe DM, Howley PM. Editors. Fields virology. Philadelphia, PA: Lippincott Williams \& Wilkins; 2007;1647-1689.

[14] Wang C, Takeuchi K, Pinto LH, Lamb RA. Ion channel activity of influenza $A$ virus $M 2$ protein: characterization of the amantadine block. J. Virol 1993;67(9):5585-5594.

[15] Lipatov AS, Govorkova EA, Webby RJ, Ozaki H, Peiris M, Guan Y, et al. Influenza: emergence and control. J Virol 2004;78(17):8951-8959. doi:10.1128/JVI.78.17.8951-8959.2004.

[16] Sheu TG, Fry AM, Garten RJ, Deyde VM, Shwe T, Bullion L, et al. Dual resistance to adamantanes and oseltamivir among seasonal influenza A(H1N1) viruses: 2008-2010. J Infect Dis 2011;203:13-17. doi:10.1093/infdis/jiq005.

[17] Hayden FG, Hay HJ. Emergence and transmission of influenza A viruses resistant to amantadine and rimantadine. Curr Top Microbiol Immunol 1992;176:119-130. doi:10.1007/978-3-642-77011-1_8.

[18] Mendel DB, Tai CY, Escarpe PA, Li WX, Sidwell RW, Huffman JH, et al. Oral administration of a prodrug of the influenza virus neuraminidase inhibitor GS 4071 protects mice and ferrets against influenza infection. Antimicrob Agents Chemother 1998;42(3):640-646.

[19] Centers for Disease Control and Prevention (CDC) [Internet]. What you should know about flu antiviral drugs. Seasonal influenza (flu) treatment - antiviral drugs. c2016 [cited 2016 September 25]. Available from: http://www.cdc.gov/flu/antivirals/whatyoushould.htm.

[20] Centers for Disease Control and Prevention (CDC) [Internet]. Antiviral drug resistance among influenza viruses. Seasonal influenza (flu) health professionalsantiviral drugs. c2015 [cited 2016 September 25]. Available from: http://www.cdc.gov/flu/professionals/antivirals/antiviral-drug-resistance.htm.

[21] Le QM, Wertheim HF, Tran ND, van Doorn HR, Nguyen TH, Horby $\mathrm{P}$, et al. A community cluster of oseltamivir-resistant cases of 2009 H1N1 influenza. N Engl J Med 2010;362(1):86-87. doi:10.1056/NEJMc0910732.

[22] Centers for Disease Control and Prevention (CDC). Oseltamivirresistant novel influenza $A(H 1 N 1)$ virus infection in two immunosuppressed patients-Seattle, Washington, 2009. MMWR 2009;58(32):893.

[23] Centers for Disease Control and Prevention (CDC). Oseltamivirresistant 2009 pandemic influenza $A$ (H1N1) virus infection in two summer campers receiving prophylaxis-north Carolina-2009. MMWR 2009;58(35):969-972.

[24] Inoue M, Barkham T, Leo YS, Chan KP, Chow A, Wong CW, et al. Emergence of oseltamivir-resistant pandemic (H1N1) 2009 virus within 48 hours. Emerg Infect Dis 2010;16(10):1633-1636. doi:10.3201/ eid1610.100688.

[25] Hatakeyama S, Sugaya N, Ito M, Yamazaki M, Ichikawa M, Kimura K, et al. Emergence of influenza B viruses with reduced sensitivity to neuraminidase inhibitors. JAMA 2007;297(13):1435-1442. doi:10.1001/ jama.297.13.1435.

[26] Gubareva LV, Matrosovich MN, Brenner MK, Bethell RC, Webster RG. Evidence for zanamivir resistance in an immunocompromised child infected with influenza B virus. J Infect Dis 1998;178(5):1257-1262. doi:10.1086/314440.

[27] Ison MG, Gubareva LV, Atmar RL, Treanor J, Hayden FG. Recovery of drug-resistant influenza virus from immunocompromised patients: a case series. J Infect Dis 2006;193(6):760-764. doi:10.1086/500465.

[28] Stephenson I, Democratis J, Lackenby A, McNally T, Smith J, Pareek M et al. Neuraminidase inhibitor resistance after oseltamivir treatment of acute influenza A and B in children. Clin Infect Dis 2009;48(4):389396. doi:10.1086/596311.

[29] Stein DS, Creticos CM, Jackson GG, Bernstein JM, Hayden FG, Schiff GM, et al. Oral ribavirin treatment of influenza A and B. Antimicrob Agents Chemother. 1987;31(8):1285-1287. doi:10.1128/ AAC.31.8.1285.

[30] Beigel J, Bray M. Current and future antiviral therapy of severe seasonal and avian influenza. Antivir Res 2008;78:91-102. doi:10.1016/j. antiviral.2008.01.003.

[31] Chan-Tack KM, Murray JS, Birnkrant DB. Use of ribavirin to treat influenza. N Engl J Med 2009;361(17):1713-1714. doi:10.1056/NEJMc0905290.

[32] Nguyen JT, Hoopes JD, Le MH, Smee DF, Patick AK, Faix DJ, et al. Triple combination of amantadine, ribavirin, and oseltamivir is highly active and synergistic against drug resistant influenza virus strains in vitro. PLoS ONE 2010;5(2):e9332. doi:10.1371/journal.pone.0009332.

[33] Seo S, Englund JA, Nguyen JT, Pukrittayakamee S, Lindegardh N, Tarning J, et al. Combination therapy with amantadine, oseltamivir and ribavirin for influenza A infection: safety and pharmacokinetics. Antivir Ther 2013;18(3):377-386. doi:10.3851/IMP2475.

[34] Kim WY, Young SG, Huh JW, Kim SH, Kim MJ, Kim YS, et al. Triple-combination antiviral drug for pandemic H1N1 influenza virus infection in critically III Patients on mechanical ventilation. Antimicrob Agents Chemother 2011;55:5703-5709. doi:10.1128/AAC.05529-11.

[35] Sanjuán R, Nebot MR, Chirico N, Mansky LM, Belshaw R. Viral mutation rates. J Virol 2010;84:9733-9748. doi:10.1128/JVI.00694-10.

[36] Steinhauer DA, Domingo E, Holland JJ. Lack of evidence for proofreading mechanisms associated with an RNA virus polymerase. Gene 1992;122(2):281-288. doi:10.1016/0378-1119(92)90216-C.

[37] Elena SF, Sanjuan R. Adaptive Value of High Mutation Rates of RNA viruses: separating causes from consequences. J Virol 2005; 79(18):11555-11558. doi:10.1128/JVI.79.18.11555-11558.2005.

[38] Ison MG, Gnann JW Jr, Nagy-Agren S, Treannor J, Paya C, Steigbigel $\mathrm{R}$, et al. Safety and efficacy of nebulized zanamivirin hospitalized patients with serious influenza. Antivir Ther 2003;8(3):183-190.

[39] Visher E, Whitefield SE, McCrone JT, Fitzsimmons W, Lauring AS. The mutational robustness of influenza A virus. PLoS Pathog 2016;12(8):e1005856. doi:10.1371/journal.ppat.1005856.

[40] Hurt AC, Holien JK, Parker MW, Barr IG. Oseltamivir resistance and the $\mathrm{H} 274 \mathrm{Y}$ neuraminidase mutation in seasonal, pandemic and highly pathogenic influenza viruses. Drugs 2009;69:2523-2531. doi:10.2165/11531450-000000000-00000.

[41] Meijer A, Lackenby A, Hungnes O, Lina B, van-der-Werf S, Schweiger $B$, et al. Oseltamivir-resistant influenza virus A (H1N1), Europe, 2007-08 Season. Emerg Infect Dis 2009;15:552-560. doi:10.3201/ eid1504.081280.

[42] Richard M, Ferraris O, Erny A, Barthélémy M, Traversier A, Sabatier $M$, et al. Combinatorial effect of two framework mutations (E119V and $1222 \mathrm{~L}$ ) in the neuraminidase active site of H3N2 influenza virus on resistance to oseltamivir. Antimicrob Agents Chemother 2011;55:2942-2952. doi:10.1128/AAC.01699-10.

[43] Barik S. New treatments for influenza. BMC Medicine 2012;10:104. doi:10.1186/1741-7015-10-104.

[44] Wolf YI, Viboud C, Holmes EC, Koonin EV, Lipman DJ. Long intervals of stasis punctuated by bursts of positive selection in the seasonal evolution of influenza A virus. Biol Direct 2006;1:34. doi:10.1186/17456150-1-34.

[45] Centers for Disease Control and Prevention (CDC) [Internet]. Influenza antiviral drug resistance. c2016 [cited 2016 September 29]. Available from: http://www.cdc.gov/flu/about/qa/antiviralresistance.htm.

[46] Adeola OA, Olugasa BO, Emikpe BO. Antigenic detection of human strain of influenza virus $A(H 3 N 2)$ in swine populations at three locations in Nigeria and Ghana during the dry early months of 2014. Zoonose Public Health 2016;63(2):106-111. doi:10.1111/zph.12210.

[47] Ayora-Talavera G, Cadavieco-Burgos JM, Canul-Armas AB. Serologic evidence of human and swine influenza in Mayan persons. Emerg Inf Dis 2005;11(1):158-160. doi:10.3201/eid1101.040554.

[48] World Health Organization (WHO) [Internet]. Influenza (Seasonal). Fact sheet No 211, March 2014. c2014 [cited 2016 September 25]. Available from: http://www.who.int/mediacentre/factsheets/fs211/en/.

[49] Adeola OA, Olugasa BO, Emikpe BO. Detection of pandemic strain of influenza virus (A/H1N1/pdm09) in pigs, West Africa: implications and considerations for prevention of future influenza pandemics at the source. Infect Ecol Epidemiol 2015;5:30227. doi:10.3402/iee. v5.30227.

[50] Centers for Disease Control and Prevention (CDC) [Internet]. Situation summary on influenza A (H3N2) variant viruses ("H3N2v"). c2016. [cited 2016 September 27]. Available from: http://www.cdc. gov/flu/swineflu/h3n2v-situation.htm.

[51] Epperson S, Jhung M, Richards S, Quinlisk P, Ball L, Moll M, et al. Human infections with influenza $A(H 3 N 2)$ variant virus in the Unit- 
ed States, 2011-2012. Clin Infect Dis 2013;57(Suppl 1):S4-S11. doi: $10.1093 / \mathrm{cid} / \mathrm{cit} 272$

[52] Centers for Disease Control and Prevention (CDC) [Internet]. H3N2v and you. c2014. [cited 2016 September 30]. Available from: http:// www.cdc.gov/flu/swineflu/h3n2v-basics.htm.

[53] Centers for Disease Control and Prevention (CDC) [Internet]. American Indians, Alaska natives, and the flu. CDC features: life stages \& populations. c2015a [cited 2016 September 25]. Available from: http://www.cdc.gov/features/aianflu/.

[54] Centers for Disease Control and Prevention (CDC) [Internet]. People at high risk of developing flu-related complications. Seasonal influenza (flu): specific groups. c2016b [cited 2016 September 24]. Available from: http://www.cdc.gov/flu/about/disease/high_risk.htm.

[55] Vaucheret H, Beclin C, Farard M. Post-transcriptional gene silencing in plants. J Cell Sci 2001;114(Pt 17):3083-3091.

[56] Betákova T, Svančarová P. Role and application of RNA interference in replication of influenza viruses. Acta Virol 2013;57(2):97-104. doi:10.4149/av_2013_02_97.

[57] Lee RC, Feinbaum RL, Ambros V. The C. elegans heterochronic gene lin-4 encodes small RNAs with antisense complementarity to lin-14. Cell 1993;75(5):843-854. doi:10.1016/0092-8674(93)90529-Y.

[58] Napoli C, Lemieux C, Jorgensen R. Introduction of a chimeric chalcone synthase gene into petunia results in reversible co-suppression of homologous genes in trans. Plant Cell 1990;2(4):279-289. doi:10.1105/ tpc.2.4.279.

[59] Romano N, Macino G. Quelling: transient inactivation of gene expression in Neurospora crassa by transformation with homologous sequences. Mol Microbiol 1992;6(22):3343-3353. doi:10.1111/j.1365-2958.1992.tb02202.x.

[60] Fire A, Xu S, Montgomery MK, Kostas SA, Driver SE, Mello CC. Potent and specific genetic interference by double-stranded RNA in caenorhabditis elegans. Nature 1998;391(6669):806-811. doi: $10.1038 / 35888$.

[61] Guo S, Kemphues KJ. par-1, a gene required for establishing polarity in C. elegans embryos, encodes a putative Ser/Thr kinase that is asymmetrically distributed. Cell 1995;81(4):611-620. doi:10.1016/00928674(95)90082-9.

[62] Hammond, Bernstein E, Beach D, Hannon GJ. An RNA-directed nuclease mediates post-transcriptional gene silencing in Drosophila cells. Nature 2000;404(6775):293-296. doi:10.1038/35005107.

[63] Zamore P, Tuschl T, Sharp P, Bartel D. RNAi: Double-stranded RNA directs the ATP-dependent cleavage of mRNA at 21 to 23 nucleotide intervals. Cell 2000;101(1):25-33. doi:10.1016/S0092-74(00)806200 .

[64] Bernstein E, Caudy AA, Hammond SM, Hannon GJ. Role for a bidentate ribonuclease in the initiation step of RNA interference. Nature 2001;409:363-366. doi:10.1038/35053110.

[65] Bitko V, Barik S. Phenotypic silencing of cytoplasmic genes using sequence-specific double-stranded short interfering RNA and its application in the reverse genetics of wild type negative-strand RNA viruses. BMC Microbiology 2001;1:34. doi:10.1186/1471-2180-1-34.

[66] Ge Q, McManus MT, Nguyen T, Shen CH, Sharp PA, Eisen HN, et al. RNA interference of influenza virus production by directly targeting mRNA for degradation and indirectly inhibiting all viral RNA transcription. PNAS 2003;100(5):2718-2723. doi:10.1073/pnas.0437841100.

[67] Song JJ, Smith SK, Hannon GJ, Joshua-Tor L. Crystal structure of Argonaute and its implications for RISC slicer activity. Science 2004;305(5689):1434-1437. doi:10.1126/science.1102514.

[68] Martinez J, Tuschl T. RISC is a $5^{\prime}$ phosphomonoester-producing RNA endonuclease. Genes Dev 2004;18(9):975-980. doi:10.1101/ gad.1187904.

[69] Liu J, Carmell MA, Rivas FV, Marsden CG, Thomson JM, Song JJ, et al. Argonaute2 is the catalytic engine of mammalian RNAi. Science 2004;305(5689):1437-1441. doi:10.1126/science.1102513.

[70] Nobel Media AB 2014 [Internet]. Press Release: The 2006 Nobel Prize in Physiology or Medicine. c2016 [cited 2016 October 11]. Available from: http://www.nobelprize.org/nobel_prizes/medicine/laureates/ 2006/press.html.

[71] Agrawal N, Dasaradhi PVN, Mohmmed A, Malhotra P, Bhatnagar RK, Mukherjee SK. RNA interference: biology, mechanism, and applications. Microbiol Mol Biol Rev 2003;67(4):657-685. doi:10.1128/
MMBR.67.4.657-685.2003.

[72] Jinek M, Doudna JA. A Three-dimensional view of the molecular machinery of RNA interference. Nature 2009;457(7228):405-412. doi:10.1038/nature07755.

[73] MacFarlane LA, Murphy PR. MicroRNA: biogenesis, function and role in cancer. Curr Genomics 2010;11(7):537-561. doi:10.2174/ 138920210793175895.

[74] Wilson RC, Doudna JA. Molecular mechanisms of RNA interference. Annu Rev Biophys 2013;42:217-239. doi:10.1146/annurev-biophys-083012-130404.

[75] Fujita Y, Kuwano K, Ochiya T. Development of small RNA delivery systems for lung cancer therapy. Int J Mol Sci 2015;16(3):5254-5270. doi:10.3390/ijms16035254.

[76] Hutvágner G, Zamore PD. RNAi: nature abhors a double strand. Curr Opin Genet Dev 2002;12(2):225-232. doi:10.1016/S0959-437X (02)00290-3.

[77] McMannus MT, Sharp PA. Gene silencing in mammals by small interfering RNAs. Nat Rev Genet 2002;3(10):737-747. doi:10.1038/ nrg908.

[78] Doi N, Zenno S, Ueda R, Ohki-Hamazaki H, Ui-Tei K, Saigo K. Short-interfering-RNA-mediated gene silencing in mammalian cells requires dicer and elF2C translation initiation factors. Curr Biol 2003;13(1):4146. doi:10.1016/S0960-9822(02)01394-5.

[79] Sui HY, Zhao GY, Huang JD, Jin DY, Yuen KY, Zheng BJ. Small interfering RNA targeting $M 2$ gene induces effective and long term inhibition of influenza A virus replication. PLoS ONE 2009;4(5):e5671. doi:10.1371/journal.pone.0005671.

[80] Carthew RW, Sontheimer EJ. Origins and Mechanisms of miRNAs and siRNAs. Cell 2009;4(5):e5671. doi:10.1371/journal.pone.0005671.

[81] Brummelkamp TR, Bernards R, Agami R. A system for stable expression of short interfering RNAs in mammalian cells. Science 2002;296(5567):550-3. doi:10.1126/science.1068999.

[82] Paddison PJ, Caudy AA, Bernstein E, Hannon GJ, Conklin DS. Short hairpin RNAs (shRNAs) induce sequence-specific silencing in mammalian cells. Genes Dev 2002;16(8):948-58. doi:10.1101/gad.981002.

[83] Wang Z, Rao DD, Senzer N, Nemunaitis J. RNA interference and cancer therapy. Pharm Res 2011;28(12):2983-2995. doi:10.1007/ s11095-011-0604-5.

[84] Elbashir SM, Harborth J, Lendeckel W, Yalcin A, Weber K, Tuschl T. Duplexes of 21-nucleotide RNAs mediate RNA interference in cultured mammalian cells. Nature 2001;411(6836):494-498. doi:10.1038/35078107.

[85] Aagaard L, Rossi JJ. RNAi therapeutics: principles, prospects and challenges. Adv Drug Deliv Rev 2007;59(2-3):75-86. doi:10.1016/j. addr.2007.03.005.

[86] De Fougerolles A, Vornlocher HP, Maraganore J, Lieberman J. Interfering with disease: A progress report on siRNA-based therapeutics. Nat Rev Drug Deliv 2007;6(6):443-453. doi:10.1038/nrd2310.

[87] Sugrue RJ, Tan BH, Yeo DS, Sutejo R. Antiviral drugs for the control of pandemic influenza virus. Ann Acad Med Singapore 2008;37(6):518524.

[88] Khanna M, Saxena L, Rajput R, Kumar B, Prasad R. Gene silencing: a therapeutic approach to combat influenza virus infections. Future Microbiol 2015;10(1):131-140. doi:10.2217/fmb.14.94.

[89] Barik S. siRNA for influenza therapy. Viruses 2010;2:1448-1457. doi:10.3390/v2071448.

[90] Kumar P, Kumar B, Rajput R, Saxena L, Banerjea AC, Khanna M. Crossprotective effect of antisense oligonucleotide developed against the common $3^{\prime}$ NCR of influenza A virus genome. Mol Biotechnol 2013;55(3):203-211. doi:10.1007/s12033-013-9670-8.

[91] Tompkins SM, Lo CY, Tumpey TM, Epstein SL. Protection against lethal influenza virus challenge by RNA interference in vivo. Proc Natl Acad Sci U S A 2004;101(23):8682-8686. doi:10.1073/pnas.0402630101.

[92] Garcia-Sastre A. Mechanisms of inhibition of the host interferon alpha/beta-mediated antiviral responses by viruses. Microbes Infect 2002;4(6):647-655. doi:10.1016/S1286-4579(02)01583-6.

[93] Katze MG, He Y, Gale M Jr. Viruses and interferon: a fight for supremacy. Nat Rev Immunol 2002;2(9):675-687. doi:10.1038/nri888.

[94] Behera P, Nagarajan S, Murugkar HV, Kalaiyarasu S, Prakash A Gothalwal RZ, et al. siRNAs targeting PB2 and NP genes potentially inhibit replication of highly pathogenic H5N1 avian influenza virus. J 
Biosci 2015;40(2):233-240. doi:10.1007/s12038-015-9524-6.

[95] Bitko V, Musiyenko A, Shulyayeva O, Barik S. Inhibition of respiratory viruses by nasally administered siRNA. Nat Med 2004;11(1):50-55. doi:10.1038/nm1164.

[96] Zhang W, Yang H, Kong X, Mohapatra S, San Juan-Vergara H, Hellermann $G$, et al. Inhibition of respiratory syncytial virus infection with intranasal siRNA nanoparticles targeting the viral NS1 gene. Nat Med 2005;11(1):56-62. doi:10.1038/nm1174.

[97] Jiao H, Du L, Hao Y, Cheng Y, Luo J, Kuang W, et al. Effective inhibition of mRNA accumulation and protein expression of H5N1 avian influenza virus NS1 gene in vitro by small interfering RNAs. Folia Microbiol (Praha) 2013;58(4):335-342. doi:10.1007/s12223-012-0212-8.

[98] Rajput R, Khanna M, Kumar P, Kumar B, Sharma S, Gupta N, et al. siRNA targeting the nonstructural gene transcript inhibits influenza $A$ virus replication in experimental mice. Nucleic Acid Ther 2012;22(6):414-422. doi:10.1089/nat.2012.0359.

[99] Zhou K, He H, Wu Y, Duan M. RNA interference of avian influenza virus H5N1 by inhibiting viral mRNA with siRNA expression plasmids. J Biotechnol 2008;135(2):140-144. doi:10.1016/j.jbiotec.2008.03.007.

[100] McManus MT, Sharp PA. Gene silencing in mammals by small interfering RNAs. Nat Rev Genet 2002;3(10):737-747. doi:10.1038/ nrg908.

[101] Kumar M, Carmichael GG. Antisense RNA: Function and fate of duplex RNA in cells of higher eukaryotes. Microbiol Mol Biol Rev 1998;62(4):1415-1434.

[102] Tripp RA, Tompkins SM. Johnson PH. Application of RNA Interference to viral disease. RNA interference: application to drug discovery and challenges to pharmaceutical development 2011;John Wiley and Sons Inc.328.

[103] Coburn GA, Cullen BR. siRNAs: a new wave of RNA-based therapeutics. J Antimicrob Chemother 2003;51(4):753-756. doi:10.1093/ $\mathrm{jac} / \mathrm{dkg} 166$.

[104] Belser JA, Katz JM, Tumpey TM. The ferret as a model organism to study influenza A virus infection. Dis Model Mech 2011;4(5):575579. doi:10.1242/dmm.007823.

[105] Matsuoka Y, Lamirande EW, Subbarao K. The Ferret Model for Influenza. Current Protocols in Microbiology 2009;13:G:15G.2:15G.2.115G.2.29. doi:10.1002/9780471729259.mc15g02s13.

[106] Jackson AL, Burchard J, Schelter J, Chau BN, Cleary M, Lim L, et al. Widespread siRNA "off-target" transcript silencing mediated by seed region sequence complementarity. RNA 2006;12(7):11791187. doi:10.1261/rna.25706.

[107] Jackson AL, Bartz SR, Schelter J, Kobayashi SV, Burchard J, Mao M, et al. Expression profiling reveals off-target gene regulation by RNAi. Nat Biotechnol 2003;21(6):635-637. doi:10.1038/nbt831.

[108] Jackson AL, Linsley PS. Noise amidst the silence: off-target effects of siRNAs?. Trends Genet 2004;20(11):521-524. doi:10.1016/j. tig.2004.08.006

[109] Lin X, Ruan X, Anderson MG, McDowell JA, Kroeger PE, Fesik SW, et al. siRNA-mediated off-target gene silencing triggered by a 7 nt complementation. Nucleic Acids Res 2005;33(14):4527-4535. doi:10.1093/nar/gki762.

[110] Fedorov Y, Anderson EM, Birmingham A, Reynolds A, Karpilow J, Robinson $\mathrm{K}$, et al. Off-target effects by siRNA can induce toxic phenotype. RNA 2006;12(7):1188-1196. doi:10.1261/rna.28106.

[111] Caffrey DR, Zhao J, Song Z, Schaffer ME, Haney SA, Subramanian $R R$, et al. siRNA off-target effects can be reduced at concentrations that match their individual potency. PLoS ONE 2011;6(7):e21503. doi:10.1371/journal.pone.0021503.

[112] Scacheri PC, Rozenblatt-Rosen O, Caplen NJ, Wolfsberg TG, Umayam $\mathrm{L}$, Lee JC, et al. Short interfering RNAs can induce unexpected and divergent changes in the levels of untargeted proteins in mammalian cells. Proc Natl Acad Sci U S A 2004;101(7):1892-1897. doi:10.1073/pnas.0308698100.

[113] Birmingham A, Anderson EM, Reynolds A, Ilsley-Tyree D, Leake D, Fedorov $\mathrm{Y}$, et al. 3' UTR seed matches, but not overall identity, are associated with RNAi off-targets. Nat Methods 2006;3(3):199-204. doi:10.1038/nmeth854.

[114] Semizarov D, Frost L, Sarthy A, Kroeger P, Halbert DN, Fesik SW. Specificity of short interfering RNA determined through gene expression signatures. Proc Natl Acad Sci U S A 2003;100(11):63476352. doi:10.1073/pnas.1131959100.

[115] Jackson AL, Burchard J, Leake D, Reynolds A, Schelter J, Guo J, et al. Position-specific chemical modification increases specificity of siRNA-mediated gene silencing. RNA 2006;12(7):1197-1205. doi: 10.1261/rna.30706

[116] Dua P, Yoo JW, Kim S, Lee DK. Modified siRNA structure with a single nucleotide bulge overcomes conventional siRNA-mediated offtarget silencing. Mol Ther 2009;19(9):1676-1687. doi:10.1038/ mt.2011.109.

[117] Naito Y, Ui-Tei K. siRNA design software for a target gene-specific RNA interference. Front Genet 2012;3:102. doi:10.3389/ fgene.2012.00102.

[118] Chen X, Liu P, Chou HH. Whole-genome thermodynamic analysis reduces siRNA off-target effects. PLoS ONE 2013;8(3):e58326. doi:10.1371/journal.pone.0058326.

[119] Adams R, Nicke B, Pohlenz H-D, Sohler F. Deciphering seed sequence based off-target effects in a large-scale RNAi reporter screen for Ecadherin expression. PLoS ONE 2015;10(9):e0137640. doi:10.1371/ journal.pone.0137640.

[120] Yilmazel B, Hu Y, Sigoillot F, Smith JA, Shamu CE, Perrimon N, et al Online GESS: prediction of miRNA-like off-target effects in largescale RNAi screen data by seed region analysis. BMC Bioinformat 2014;15:192. doi:10.1186/1471-2105-15-192.

[121] Torrecilla J, Rodríguez-Gascón A, Solinís MÁ, del Pozo-Rodríguez A. Lipid nanoparticles as carriers for RNAi against viral infections: current status and future perspectives. Biomed Res Int 2014;2014:161794. doi:10.1155/2014/161794.

[122] Esteban-Fernández de Ávila B, Angell C, Soto F, Lopez-Ramirez MA Báez DF, Xie $S$, et al. Acoustically propelled nanomotors for intracelIular siRNA delivery. ACS Nano 2016;10(5):4997-5005. doi:10.1021/ acsnano.6b01415. 